\title{
EFFECT OF ARONIA MELANOCARPA FRUIT JUICE ON INDICES OF INFLAMMATION AND FIBROSIS IN A RAT MODEL OF AMIODARONE-INDUCED PNEUMOTOXICITY
}

\author{
Valcheva-Kuzmanova S. ${ }^{1}$, G. Stavreva ${ }^{2}$, V. Dancheva ${ }^{3},{ }_{3}$ L. Terziev $^{4}$, M. Atanasova ${ }^{5}$, \\ A. Stoyanova ${ }^{6}$, V. Shopova \\ ${ }^{1}$ Department of Preclinical and Clinical Pharmacology and Toxicology, Medical University of \\ Varna, ${ }^{2}$ Department of Experimental and Clinical Pharmacology, Medical University of Pleven, \\ ${ }^{3}$ Department of Disaster Medicine, Medical University of Pleven, ${ }^{4}$ Clinic of Allergology, University \\ Hospital of Pleven, ${ }^{5}$ Department of Biology, Medical University of Pleven, \\ ${ }^{6}$ Department of Chemistry, Medical University of Pleven
}

\begin{abstract}
The effect of Aronia melanocarpa fruit juice (AMFJ) on indices of inflammation and fibrosis was studied in a model of amiodarone (AD)-induced pneumotoxicity in rats. AD was instilled intratracheally on days 0 and 2 $(6,25 \mathrm{mg} / \mathrm{kg}$ as a $3,125 \mathrm{mg} / \mathrm{mL}$ water solution). AMFJ $(10 \mathrm{~mL} / \mathrm{kg})$ was given orally to rats either from day 1 to day 10, or from day 11 to day 27. Thus, the animal groups were: control, AD, AD+AMFJ (day 1-10), and $A D+A M F J$ (day 11-27). The rats were sacrificed on day 28 . The levels of IL-6 and IL-10 were measured in rat serum as markers of inflammation, and hydroxyproline (HP) level was determined in lung tissue as a marker of fibrosis. AD caused a tendency to elevate IL-6 and decrease IL-10. AMFJ counteracted these effects of AD. In rats from group AD+AMFJ (day 1-10), IL-6 level was significantly lower $(p<0,05)$ than that of AD group, lower $(p<0,05)$ even than the control value. AD significantly increased $(p<0,05)$ HP content in lung homogenate. AMFJ antagonized that effect, and in AMFJ-treated rats HP levels did not differ significantly from the control value. Any AMFJ effects were more prominent in rats that were treated with the juice during the first 10 days after AD instillation. In conclusion, AMFJ reduced the signs of inflammation and could have a protective effect against AD-induced pulmonary fibrosis, especially if administered in the early phase after AD instillation.
\end{abstract}

Key words: Aronia melanocarpa fruit juice, amiodarone, lung, inflammation, fibrosis, rats

\section{INTRODUCTION}

Amiodarone $(\mathrm{AD})$ is a very effective antiarrhythmic drug. It causes acute pneumonitis resulting in fatal pulmonary fibrosis (8). In vivo and in vitro studies have shown that $\mathrm{AD}$ is not only directly toxic to lung cells (16) but also could induce oxidative stress and increased production of reactive oxygen species $(11,19)$, activation of alveolar macrophages and cytokine release $(4,14,20)$.

Aronia melanocarpa (Michx.) Elliot (black chokeberry) fruits are extremely rich in phenolic compounds (12): procyanidins, flavonoids (mainly from the subclass of anthocyanins) and phenolic acids (chlorogenic and neochlorogenic). Aronia berries possess very high antioxidant capacity $(9,12,18)$. Studies have demonstrated that constituents of Aronia melanocarpa fruits (flavonoids in-

\footnotetext{
Address for correspondence

S. Valcheva-Kuzmanova, Dept. of Preclinical and Clinical

Pharmacology and Toxicology, Medical University of Varna,

55, Marin Drinov Str., 9002 Varna, Bulgaria

e-mail: stefkavk@yahoo.com
}

cluding anthocyanins) possess anti-inflammatry activity due to the suppression of the release of proinflammatory cytokines such as tumor necrosis factor alpha and interleukins (IL-1beta, IL-6, IL-8) $(7,17)$.

The aim of the study was to investigate the effect of Aronia melanocarpa fruit juice (AMFJ) on indices of inflammation and fibrosis in a rat model of AD-induced pulmonary toxicity.

\section{MATERIALS AND METHODS}

\section{Experimental substances}

Amiodarone hydrochloride $(\mathrm{AD})$ and all other chemicals and reagents were of analytical grade and were purchased from Sigma-Aldrich Company (Germany). The Quantikine Rat IL-6 and IL-10 immunoassay kits were from R\&D Systems (USA).

AMFJ was produced from Aronia melanocarpa Elliot fruits grown in the Balkan Mountains, Bulgaria. They were handpicked in September, crushed and squeezed. The juice 
was filtered, pasteurized at $80^{\circ} \mathrm{C}$ for $10 \mathrm{~min}$ and stored at 0 ${ }^{\circ} \mathrm{C}$ till the experiment. The contents of phenolic substances in $100 \mathrm{~mL}$ AMFJ were: total phenolics, $709,3 \pm 28,1 \mathrm{mg}$ as gallic acid equivalents; total flavonoids, $189,4 \pm 8,6 \mathrm{mg}$ as catechin equivalents; total anthocyanins, $106,8 \pm 6,2 \mathrm{mg}$ as cyanidin-3-glucoside equivalents, and quercetin, $11,8 \mathrm{mg}$. The values were the mean of duplicate determinations of three samples.

\section{Animals and experimental treatments}

The study was carried out on 24 male Wistar rats (weight 220-250 g, age 4 months). The animals were housed at a temperature of $22 \pm 2{ }^{\circ} \mathrm{C}$ and humidity of $50 \pm 10 \%$, given normal pelleted diet and water ad libitum.

All the procedures concerning animal treatment and experimentation were conducted in compliance with the national laws and policies, in conformity with the international guidelines (EEC Council Directive 86/609, IL 358, 1, December 12, 1987).

The animals were divided into four groups of 6 rats: control, $\mathrm{AD}, \mathrm{AD}+\mathrm{AMFJ}$ (day 1-10) and AD+AMFJ (day 11-27). The control group received two intratracheal (i.t.) instillations of sterile distilled water $(2 \mathrm{~mL} / \mathrm{kg})$ on days 0 and 2 , and distilled water $(10 \mathrm{~mL} / \mathrm{kg})$ orally through an orogastric cannula from day 1 to day 10 . The $\mathrm{AD}$ group received two i.t. instillations of $\mathrm{AD}(6,25 \mathrm{mg} / \mathrm{kg}$, as a 3,125 $\mathrm{mg} / \mathrm{mL}$ water solution) on days 0 and $2(15)$, and distilled water $(10 \mathrm{~mL} / \mathrm{kg})$ orally through an orogastric cannula from day 1 to day 10 . The rats belonging to $A D+A M F J$ (day 1-10) group were treated with $\mathrm{AD}$ i.t. on days 0 and 2, and with AMFJ orally at a dose of $10 \mathrm{~mL} / \mathrm{kg}$ from day 1 to

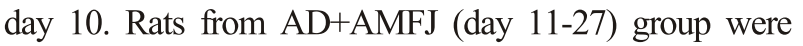
treated with $\mathrm{AD}$ i.t. on days 0 and 2 , and with $\mathrm{AMFJ}$ orally at a dose of $10 \mathrm{~mL} / \mathrm{kg}$ from day 11 to day 27 . The animals were sacrificed on day 28 under thiopental anesthesia (50 $\mathrm{mg} / \mathrm{kg}$ ) by exsanguination through cutting $v$. renalis.

$\mathrm{AD}$ was dissolved in distilled water at $60^{\circ} \mathrm{C}$ and allowed to cool to room temperature before the i.t. instillation.

\section{Immunological assays of rat serum}

The serum of the experimental animals was used for the measurement of IL- 6 and IL-10 in $\mathrm{pg} / \mathrm{mL}$ by the ELISA method in accordance with the immunoassay kits manufacturer's instructions.

\section{Biochemical assays of lung homogenate}

Lung homogenate was obtained from the right lung. The tissue was homogenized with $\mathrm{KCl}(1,15 \%)$ in 1:10 ratio. The homogenate was centrifuged $(9000 \times \mathrm{g}, 30 \mathrm{~min})$ and the supernatant was stored on ice. Hydroxyproline (HP) levels were measured in $\mathrm{Mg} / \mathrm{mL}$ as described by Bergman and Loxley (2). The method is based on the release of free HP from collagen by acid hydrolysis.

\section{Statistical analysis}

Results are presented as mean \pm S.E.M. The data were tested by one-way ANOVA, followed by Dunnett's multiple comparison post test. A level of $\mathrm{p}<0,05$ was considered significant. All analyses were performed using GraphPad Prism statistical software.

\section{RESULTS}

\section{Immunological assays of rat serum}

$\mathrm{AD}$ instillation resulted in a slight elevation of IL-6 in rat serum (104\% of the control) (Fig. 1). AMFJ prevented $\mathrm{AD}$-induced elevation of IL-6. IL-6 in rats from $\mathrm{AD}+\mathrm{AMFJ}$ (day 1-10) group was $87 \%$ of the control value, and in rats from $\mathrm{AD}+\mathrm{AMFJ}$ (day 11-27) group it was $91 \%$ of the control level. Thus, in animals belonging to $\mathrm{AD}+\mathrm{AMFJ}$ (day 1-10) group, IL-6 was significantly lower than that of $A D$ group $(p<0,05)$. It was significantly lower even than the control value $(\mathrm{p}<0,05)$ (Fig. 1).

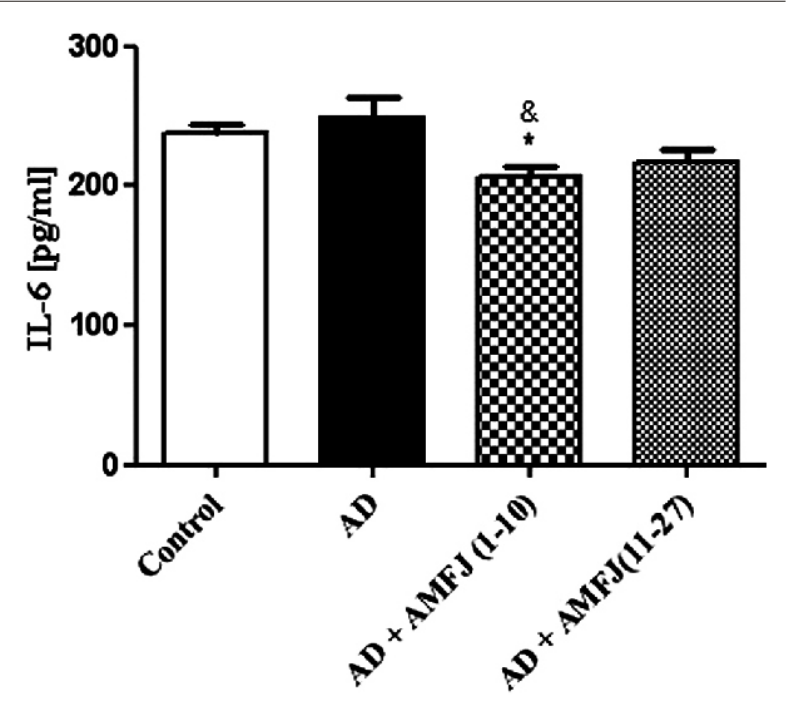

Fig. 1. Effect of AMFJ on IL-6 levels in rat serum 28 days after i.t. $A D$ instillation; $A M F J$ applied with $A D$ either from day 1 to day 10, or from day 11 to day 27 ${ }^{*} p<0,05$ vs control; ${ }_{p}^{\mathrm{d}}<0,05$ vs $A D$

In $\mathrm{AD}$ group, a tendency was observed for reduction of IL-10 to a level that was $91 \%$ of the control value (Fig. 2). AMFJ antagonized that effect of AD. In AMFJ-treated animals, IL-10 levels were higher (however, insignificantly) not only than the $\mathrm{AD}$ group level but also than the control one. Thus, IL-10 levels were $108 \%$ of the control value for $\mathrm{AD}+\mathrm{AMFJ}$ (day 1-10) group and $106 \%$ of the control value for $\mathrm{AD}+\mathrm{AMFJ}$ (day 11-27) group (Fig. 2).

\section{Biochemical assays of lung homogenate}

$\mathrm{HP}$ levels are presented on Fig. 3. In rats from $\mathrm{AD}$ group, there was a significant increase ( $<<0,05$ vs control) of HP levels in lung tissue (147\% of the control value). In rats from $\mathrm{AD}+\mathrm{AMFJ}$ (day 1-10) group, HP was $112 \%$ of the control value, and in rats from AD+AMFJ (day 11-27) group, HP was $129 \%$ of the control value. Thus, in rats with $\mathrm{AD}$ instillation and treatment with AMFJ, the HP levels did not differ significantly from the control value. 


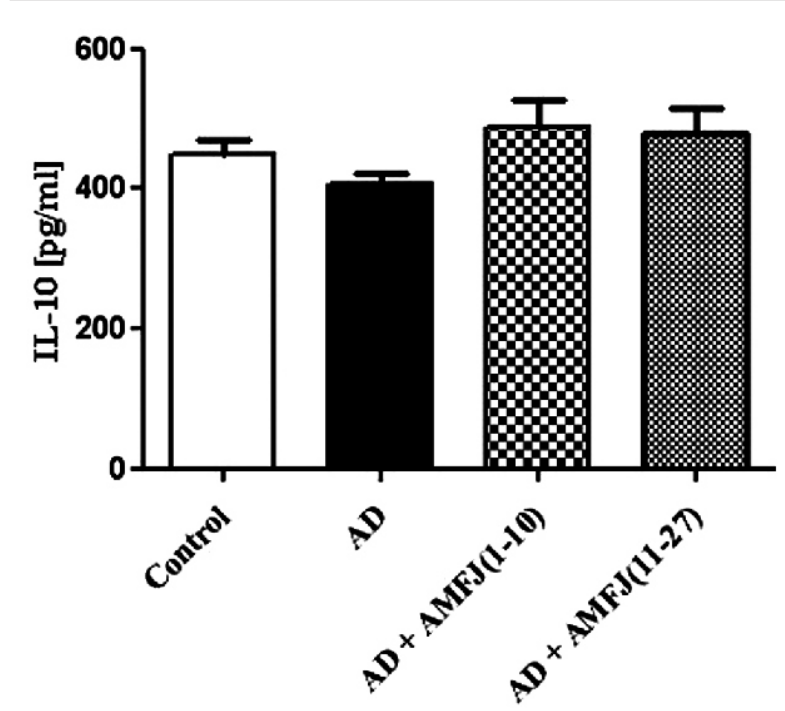

Fig. 2. Effect AMFJ on IL-10 levels in rat serum 28 days after i.t. AD instillation; AMFJ applied with AD either from day 1 to day 10, or from day 11 to day 27

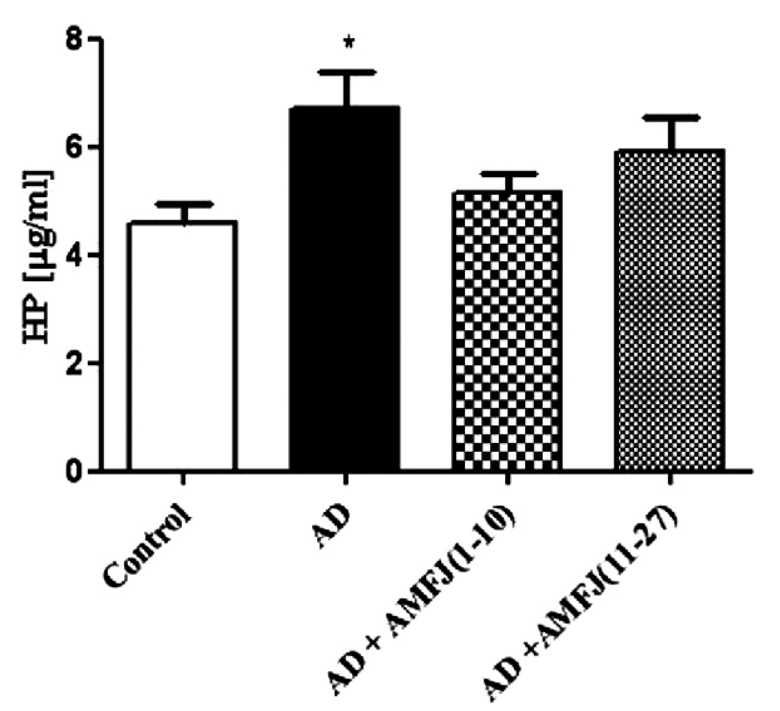

Fig. 3. Effect of AMFJ on HP levels in rat lung tissue 28 days after i.t. AD instillation; AMFJ applied with AD either from day 1 to day 10, or from day 11 to day 27 ${ }^{*} p<0,05$ vs control

\section{DISCUSSION}

Both indirect and direct toxic effects on target cells have been proposed for explanation of AD-induced pulmonary toxicity. There are findings that activation of macrophages and release of inflammatory and cytotoxic mediators drive $\mathrm{AD}$-induced lung fibrosis $(4,14)$. Up to date, there are no literature data on the effect of $\mathrm{AD}$ on the levels of the cytokines IL-6 and IL-10 available.

IL-6 is a potent, pleiotropic, inflammatory cytokine secreted by $\mathrm{T}$ cells and macrophages. It is produced at the site of inflammation and plays a key role in the acute phase re- sponse as defined by a variety of clinical and biological features such as the production of acute phase proteins. In this experiment, serum IL-6 was significantly increased in rats in the early phase (on days 3 and 5) after i.t. AD instillation (unpublished data). This could be a result of IL-6 translocation from the lung tissue to the circulation (10). The present data demonstrated that 28 days after AD instillation, serum IL-6 remained still elevated to a certain extent. It is known that when IL-6 activity as a proinflammatory cytokine persists, acute inflammation turns into chronic one that includes immune responses (6). IL-6 seems to play an important role in fibrosis initiation and progression. Rats treated with AMFJ had significantly lower IL-6 levels in comparison both with the AD and the control groups. The effect of AMFJ was more pronounced in rats treated with the juice during the first 10 days after $\mathrm{AD}$ instillation. Thus, applied in the early phase of inflammation, AMFJ could, probably, prevent the shift from acute to chronic inflammation. This effect is consistent with the anti-inflammatory activity of the flavonoids which are essential juice constituents $(7,17)$.

IL-10 is a potent anti-inflammatory cytokine. Nowadays it is known that the ability to synthesize that cytokine is not limited to certain T cell subsets, but is a characteristic of almost any leukocytes. Usually, IL-10 synthesis occurs as a consequence of acute and chronic inflammatory responses, and IL-10 neutralization often exacerbates inflammatory lesions (1). AD induced a tendency to decrease IL-10 in rat serum in the acute phase of inflammation (unpublished data), as well as in its chronic phase (current results). AMFJ antagonized that effect of $\mathrm{AD}$, which, probably, contributed to its anti-inflammatory activity.

Since lung HP is almost exclusively derived from collagen (5), whole lung collagen content was estimated by measuring HP levels. The fibrotic response in $\mathrm{AD}$ group was confirmed by a significant increase in lung HP above the control values. AMFJ applied either during the first 10 days, or from day 11 to day 27, suppressed that effect of $\mathrm{AD}$. HP levels in both rat groups treated with AMFJ did not differ significantly from the control value, however, the effect of AMFJ was more prominent in rats treated with the juice during the first 10 days after $\mathrm{AD}$ instillation.

Numerous data suggest the role of oxidative stress in amiodarone-induced lung fibrosis $(11,13,19)$. Free radical reactions have been suggested to play a contributory role in the fibrogenesis either directly or through inflammatory stimuli. Lipid peroxidation and certain lipid peroxidation products induce genetic overexpression of fibrogenic cytokines, the key molecules in the mechanisms of fibrosis, as well as increased collagen transcription and synthesis. Both events can be downregulated, at least in experimental models, by the use of antioxidants. Consequently, both catalytic and scavenger antioxidants attenuate AD-induced lung injury and fibrosis in animals (3). The ability of AMFJ to scavenge reactive oxygen species has been demonstrated by many authors using different well-established assays $(9,12,18)$. Therefore, AMFJ protective effect against $\mathrm{AD}$-induced pneumotoxicity and lung fibrosis might be at 
Effect of Aronia melanocarpa fruit juice on indices of inflammation and fibrosis in a rat model ...

least partly due to the ability of AMFJ to act as a powerful antioxidant.

\section{CONCLUSION}

AMFJ administered to rats in a model of $\mathrm{AD}$-induced pneumotoxicity reduces the signs of inflammation and fibrosis. Its protective effect is, probably, due to its antioxidant and anti-inflammatory properties.

\section{REFERENCES}

1. Avdiushko, R., D. Hongo, H. Lake-Bullock, A. Kaplan, D. Cohen. IL-10 receptor dysfunction in macrophages during chronic inflammation.- J. Leukocyte Biol., 70, 2001, No 4, 624-632.

2. Bergman, I., R. Loxley. Two improved and simplified methods for the spectrophotometric determination of hydroxyproline. - Anal. Chem., 35, 1963, No 12, 1961-1965.

3. Brian, J. D. Antioxidants as potential therapeutics for lung fibrosis.- Antioxid. Redox Signal., 10, 2008, No 2, 355-370.

4. Chung, W. H., B. M. Bennet, W. J. Racz, J. F. Brien, T. E. Massey. Induction of c-jun and TGF-beta 1 in Fischer 344 rats during amiodarone-induced pulmonary fibrosis.- Am. J. Physiol. Lung Cell. Mol. Physiol., 281, 2001, No 5, L1180-L1188.

5. Crystal, R. G. Lung collagen: definition, diversity and development.- Fed. Proc., 33, 1974, No 11, 2248-2255.

6. Gabay, C. Interleukin-6 and chronic inflammation.Arthrit. Res. Ther., 8, 2006, Suppl. 2, S3.

7. Gauliard, B., D. Grieve, R. Wilson, A. Crozier, C. Jenkins, W. D. Mullen, et al. The effects of dietary phenolic compounds on cytokine and antioxidant production by A549 cells.- J. Med. Food, 11, 2008, No 2, 382-384.

8. Gill, J., R. C. Heel, A. Fitton. Amiodarone: an overview of its pharmacological properties, and review of its therapeutic use in cardiac arrhythmias.Drugs, 43, 1992, No 1, 69-110.

9. Jakobek, L., M. Seruga, P. Krivak. The influence of interactions among phenolic compounds on the antiradical activity of chokeberries (Aronia melanocarpa).- Int. J. Food Sci. Nutr., 62, 2011, No 4, 345-352.

10. Kido, T., E. Tamagawa, N. Bai, K. Suda, H. H. Yang, Y. Li, et al. Particulate matter induces translocation of IL- 6 from the lung to the sys- temic circulation.- Am. J. Respir. Cell. Mol. Biol., 44, 2011, No 2, 197-204.

11. Nicolescu, A. C., Y. Ji, J. L. Comeau, B. C. Hill, T. Takahashi, J. F. Brien, et al. Direct mitochondrial dysfunction precedes reactive oxygen species production in amiodarone-induced toxicity in human peripheral lung epithelial HPL1A cells.- Toxicol. Appl. Pharmacol., 227, 2008, No 2, 70-79.

12. Oszmianski, J., A. Wojdylo. Aronia melanocarpa phenolics and their antioxidant activity.Eur. Food Res. Technol., 221, 2005, No 6, 809-813.

13. Pollak, P. T. Clinical organ toxicity of antiarrhythmic compounds: ocular and pulmonary manifestations.- Am. J. Cardiol., 84, 1999, No 9A, 37R-45R.

14. Reinhart, P. G., C. G. Gairola. Amiodarone-induced pulmonary toxicity in Fischer rats: release of tumor necrosis factor alpha and transforming growth factor beta by pulmonary alveolar macrophages.- J. Toxicol. Environ. Health, 52, 1997, No 4, 353-365.

15. Taylor, M. D., K. Van Dyke, L. L. Bowman, P. R. Miles, A. F. Hubbs, R. Shannon, et al. A characterization of amiodarone-induced pulmonary toxicity in F344 rats and identification of surfactant protein-D as a potential biomarker for the development of the toxicity.- Toxicol. Appl. Pharmacol., 167, 2000, No 3, 182-190.

16. Taylor, M. D., J. M. Antonini, J. R. Roberts, S. S. Leonard, X. Shi, P. M. Gannett, et al. Intratracheal amiodarone administration to F344 rats directly damages lung airway and parenchymal cells.- Toxicol. Appl. Pharmacol., 188, 2003, No 2, 92-103.

17. Tsuda, T., F. Horio, T. Osawa. Cyanidin 3-O-beta-D-glucoside suppresses nitric oxide production during a zymosan treatment in rats.- J. Nutr. Sci. Vitaminol. (Tokyo), 48, 2002, No 4, 305-310.

18. Valcheva-Kuzmanova, S., B. Blagović, S. Valić. Electron spin resonance measurement of radical scavenging activity of Aronia melanocarpa fruit juice.Pharmacogn. Mag., 8, 2012, No 30, 171-174.

19. Vereckei, A., A. Blazovics, I. Gyorgy, E. Feher, M. Toth, G. Szenasi, et al. The role of free radicals in the pathogenesis of amiodarone toxicity.- J. Cardiovasc. Electrophysiol., 4, 1993, No 2, 161-177.

20. Wilson, B. D., M. L. Lipmann. Amiodarone pulmonary toxicity in the rat is associated with increased lavage immunoglobulin and alveolar macrophages primed for increased interleukin-1 secretion.- Am. J. Respir. Cell Mol. Biol., 9, 1993, No 3, 295-299. 